H. Sato

Nagoya Math. J.

Vol. 180 (2005), 111-120

\title{
COMBINATORIAL DESCRIPTIONS OF TORIC EXTREMAL CONTRACTIONS
}

\author{
HIROSHI SATO
}

\begin{abstract}
In this paper, we give explicit combinatorial descriptions for toric extremal contractions under the relative setting, where varieties are not complete. It is well-known that the complete case is settled by using Reid's wall theory which can not be applied to the non-complete case. Therefore, we can achieve them by using the notion of extremal primitive relations. As applications, we can generalize some of Mustaţă's results related to Fujita's conjecture on toric varieties for the relative case.
\end{abstract}

\section{$\S 1$. Introduction}

The purpose of this paper is to give explicit combinatorial descriptions for extremal contractions from toric varieties.

If varieties are complete, it is well-known that the relative toric Mori theory was studied in $[R]$ and the above problem was settled completely for this case ([Ma, Chapter 14] explained $[\mathrm{R}]$ more precisely). The walls of the corresponding fan play important roles in the theory of $[R]$. We quickly review Reid's descriptions of toric extremal contraction morphisms.

Let $f: X \rightarrow Y$ be a projective toric morphism form a $\mathbb{Q}$-factorical toric $n$-fold $X=X_{\Delta}$. For an extremal ray $R \subset \mathrm{NE}(X / Y)$, let $\varphi_{R}: X \rightarrow W$ be the associated extremal contraction. If $X$ is complete, there exists a torus invariant curve $C$ on $X$ which spans $R$. $C$ corresponds to a wall, that is, an $(n-1)$-dimensional cone $w \in \Delta$. Roughly speaking, $W$ is obtained by removing such walls from $\Delta$.

However, if $X$ is not complete, $R$ is not necessarily generated by a torus invariant curve (see [FS, Example 4.3]). Thus, Reid's wall theory breaks in the first step. In this paper, we avoid this difficulty by using the notion of extremal primitive relations. This is a generalization of primitive relations

Received May 11, 2004.

2000 Mathematics Subject Classification: Primary 14M25; Secondary 14E30.

The author is partly supported by the Grant-in-Aid for JSPS Fellows, The Ministry of Education, Science, Sports and Culture, Japan. 
for smooth varieties introduced by $[\mathrm{B}]$ (see also $[\mathrm{C}]$ and $[\mathrm{S}]$ ). There always exists an extremal primitive relation corresponding to $R$. By using this relation, we can construct $W$ similarly as Reid's construction. Furthermore, the descriptions are much simpler than Reid's. Fujino's completion theorem for toric contraction morphisms in $[\mathrm{Fj} 2]$ is important for our theory.

We remark that the general theory of the relative toric Mori theory for non-complete varieties were studied in [FS]. For examples, the existence of contraction morphisms, the existence of flips and the termination of flips were studied. Thus, the combinatorial aspect of the toric Mori theory studied in this paper is a kind of supplement to [FS].

In addition, it seems very complicated to discuss the combinatorial aspect of the toric Mori theory without the $\mathbb{Q}$-factoriality. There exist various strange examples of contraction morphisms for non- $\mathbb{Q}$-factorial varieties in $[\mathrm{Fj} 2]$.

The relative toric Mori theory for non-complete varieties is completed by [Fj2], [FS] and this paper similarly as the toric Mori theory for complete varieties in $[R]$.

The content of this paper is as follows: Section 2 is a section for preparation. We review the local descriptions of toric extremal contractions when varieties are complete. We introduce the notion of extremal primitive relations. It is useful for describing toric extremal contractions. In Section 3, we give explicit descriptions for toric extremal contractions. Since we can apply Fujino's theorem, the problem becomes more elementary. In Section 4, we give some generalizations of [Mu] related to Fujita's conjecture as applications of the results in Section 3.

Notation. Here, we summarize the some notation which we will use in this paper.

(1) Let $\Delta$ be a fan. For a cone $\sigma \in \Delta$, we denote the corresponding closed orbit by $\mathrm{V}(\sigma)$.

(2) For a variety $X$ (resp. morphism $f: X \rightarrow Y$ ), we denote the Picard number (resp. relative Picard number) by $\rho(X)$ (resp. $\rho(X / Y)$ ).

(3) The symbols $\mathbb{R}_{\geq 0}, \mathbb{Z}_{>0}$ and $\mathbb{Z}_{<0}$ denote the set of non-negative real numbers, the set of positive integers and the set of negative integers, respectively. 
Acknowledgements. The author would like to thank Professor Osamu Fujino for introducing him to this problem and giving useful comments. He also wishes to thank Professor Natsuo Saito for advice and encouragement.

\section{§2. Preliminaries}

In this section, we review the toric Mori theory in $[\mathrm{R}]$. For fundamental properties of the toric geometry, see $[\mathrm{Fl}]$ and $[\mathrm{O}]$. We recommend $[\mathrm{Fj} 1],[\mathrm{FS}]$ and $[\mathrm{Ma}]$ for understanding the toric Mori theory.

Let $X=X_{\Delta}$ be a $\mathbb{Q}$-factorial toric $n$-fold associated to a fan $\Delta$ in $N=\mathbb{Z}^{n}$ over an algebraically closed field. Let $\mathrm{G}(\Delta)$ be the set of primitive generators of 1-dimensional cones in $\Delta$, and put $\mathrm{G}(\sigma):=\sigma \cap \mathrm{G}(\Delta)$ for a cone $\sigma \in \Delta$. Assume that $X$ is complete. The following notion introduced in $[\mathrm{B}]$ is useful for describing extremal rays of $X$. See $[\mathrm{B}],[\mathrm{C}]$ and $[\mathrm{S}]$ more precisely.

Definition 2.1. A non-empty subset $P \subset \mathrm{G}(\Delta)$ is a primitive collection if $P$ does not generate any cone in $\Delta$, while every proper subset of $P$ generates a cone in $\Delta$.

Remark 2.2. We can not define the primitive collection for non-simplicial fan, since for a non-simplicial cone $\sigma \in \Delta$, there exists a subset $S \subset$ $\mathrm{G}(\sigma)$ which do not generate any cone in $\Delta$.

Let $f: X \rightarrow Y$ be a projective toric morphism. For an extremal ray $R \subset \mathrm{NE}(X / Y)$, let $w \in \Delta$ be the $(n-1)$-dimensional cone corresponding to $R$. By the completeness of $X$, there exist exactly two maximal cones $\sigma_{1}, \sigma_{2} \in \Delta$ such that $w \prec \sigma_{1}$ and $w \prec \sigma_{2}$. We put $\mathrm{G}(w):=\left\{v_{1}, \ldots, v_{n-1}\right\}$, $\mathrm{G}\left(\sigma_{1}\right) \backslash \mathrm{G}(w):=\left\{v_{n}\right\}$ and $\mathrm{G}\left(\sigma_{2}\right) \backslash \mathrm{G}(w):=\left\{v_{n+1}\right\}$. After rearranging the elements in $\mathrm{G}(w)$, we obtain the equality

$$
c_{1} v_{1}+\cdots+c_{\alpha} v_{\alpha}+c_{\beta+1} v_{\beta+1}+\cdots+c_{n} v_{n}+c_{n+1} v_{n+1}=0,
$$

where $c_{1}, \ldots, c_{\alpha} \in \mathbb{Z}_{<0}, c_{\beta+1}, \ldots, c_{n+1} \in \mathbb{Z}_{>0}, \operatorname{gcd}\left(c_{1}, \ldots, c_{n+1}\right)=1$ and $\left\{v_{\beta+1}, \ldots, v_{n+1}\right\}$ is a primitive collection. We call this relation an extremal primitive relation.

Remark 2.3. In [B], [C] and [S], the arguments are developed under the assumption that varieties are smooth. However, combinatorial results of them like Proposition 2.5 hold for the simplicial case too. 
Let $\sigma_{i}$ be the cone generated by $\left\{v_{\beta+1}, \ldots, v_{n+1}\right\} \backslash\left\{v_{i}\right\}$ for $\beta+1 \leq i \leq$ $n+1$. By definition, we have $\sigma_{i} \in \Delta$. Put $w^{\prime}:=\mathbb{R}_{\geq 0} v_{1}+\cdots+\mathbb{R}_{\geq 0} v_{\alpha} \prec w$. The following proposition is one of the main results in $[R]$.

Proposition 2.4. For any cone $\sigma \in \Delta$ such that $w^{\prime} \prec \sigma$, put $\sigma=w^{\prime}+$ $\sigma^{\prime}+\tau$, where $\sigma^{\prime}, \tau \in \Delta, \mathrm{G}\left(\sigma^{\prime}\right) \subset\left\{v_{\beta+1}, \ldots, v_{n+1}\right\}$ and $\mathrm{G}(\tau) \cap\left\{v_{1}, \ldots, v_{\alpha}\right.$, $\left.v_{\beta+1}, \ldots, v_{n+1}\right\}=\emptyset$. Then, $w^{\prime}+\sigma_{i}+\tau \in \Delta$ for any $\beta+1 \leq i \leq n+1$.

Proposition 2.4 is equivalent to the following. This one is simpler, since we use the notion of primitive collections. We can confirm this equivalence as in the proof of [S, Theorem 4.10].

Proposition 2.5. ([C, Proposition 3.4] and [S, Theorem 4.10]) If $Q$ is a primitive collection of $\Delta$ such that $Q \neq\left\{v_{\beta+1}, \ldots, v_{n+1}\right\}$ and $Q \cap$ $\left\{v_{\beta+1}, \ldots, v_{n+1}\right\} \neq \emptyset$, then $\left(Q \backslash\left\{v_{\beta+1}, \ldots, v_{n+1}\right\}\right) \cup\left\{v_{1}, \ldots, v_{\alpha}\right\}$ contains $a$ primitive collection.

By using Proposition 2.4, we can construct the extremal contraction $\varphi_{R}: X \rightarrow W$ associated to $R$ (see Section 3 ).

2.6. (Extremal primitive relations) Let $f: X \rightarrow Y, R$ and $w$ as above. We rewrite the extremal primitive relations with respect to $R$ as

$$
a_{1} x_{1}+\cdots+a_{l} x_{l}=b_{1} y_{1}+\cdots+b_{m} y_{m},
$$

where $\left\{x_{1}, \ldots, x_{l}, y_{1}, \ldots, y_{m}\right\} \subset \mathrm{G}(\Delta)$ and $a_{1}, \ldots, a_{l}, b_{1}, \ldots, b_{m} \in \mathbb{Z}_{>0}$. Namely, $l=n-\beta+1, m=\alpha, a_{1} x_{1}+\cdots+a_{l} x_{l}=c_{\beta+1} v_{\beta+1}+\cdots+c_{n+1} v_{n+1}$ and $b_{1} y_{1}+\cdots+b_{m} y_{m}=-\left(c_{1} v_{1}+\cdots+c_{\alpha} v_{\alpha}\right)$. The following is obvious.

Proposition 2.7. For a torus invariant prime divisor $D$ which corresponds to $v \in \mathrm{G}(\Delta)$ and a curve $C$ which spans $R$, the following holds:

$$
(D \cdot C)\left\{\begin{array} { l } 
{ > 0 } \\
{ = 0 } \\
{ < 0 }
\end{array} \quad \text { if and only if } \quad \left\{\begin{array}{l}
v \in\left\{x_{1}, \ldots, x_{l}\right\}, \\
v \notin\left\{x_{1}, \ldots, x_{l}, y_{1}, \ldots, y_{m}\right\}, \\
v \in\left\{y_{1}, \ldots, y_{m}\right\} .
\end{array}\right.\right.
$$

We can completely recover $\Delta$ from three data: $N$, the primitive collections and the extremal primitive relations. This follows from the well-known isomorphism

$$
\mathrm{A}_{1}(X) \otimes \mathbb{Q} \cong\left\{\left(c_{x}\right)_{x \in \mathrm{G}(\Delta)} \in \mathbb{Q}^{\mathrm{G}(\Delta)} \mid \sum_{x \in \mathrm{G}(\Delta)} c_{x} x=0\right\},
$$


where $\mathrm{A}_{1}(X)$ is the Chow group of 1-cycles. In particular, if $\mathrm{G}(\Delta)$ generates $N$, then we can recover $\Delta$ from the primitive collections and the extremal primitive relations. If $\rho(X)=1$, then this condition is equivalent to the one that $X$ is a weighted projective space. This is well-known.

ExAmple 2.8. Let $X=X_{\Delta}$ be a $\mathbb{Q}$-factorial terminal toric $\mathbb{Q}$-Fano 3 -fold with Picard number 1 whose extremal primitive relation is $x_{1}+x_{2}+$ $x_{3}+x_{4}=0$. Then, there exist exactly two possibilities for such $\Delta$ (see $\left.[\mathrm{K}]\right)$.

\section{§3. Relative toric Mori theory}

In this section, we deal with the relative toric Mori theory from the combinatorial viewpoint. We remark that varieties are not necessarily complete. For the general theory, see [FS].

Remark 3.1. [Ma, Chapter 14] and [R] are good references to the toric Mori theory, too. However, in [Ma, Chapter 14] and [R], varieties are assumed to be complete. In particular, there is a meaningless statement in [Ma, Proposition 14-1-5].

Let $f: X=X_{\Delta} \rightarrow Y$ be a projective surjective toric morphism with $\operatorname{dim} X=n$. We assume that $X$ is $\mathbb{Q}$-factorial. For an extremal ray $R \subset$ $\mathrm{NE}(X / Y)$, let $\varphi:=\varphi_{R}: X \rightarrow W$ be the associated extremal contraction. The following Fujino's theorem in $[\mathrm{Fj} 2]$ is the key to the main result of this section.

TheOREM 3.2. ([Fj2, Theorem 2.10]) There exist equivariant completions

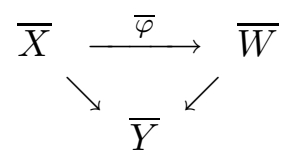

of toric morphisms

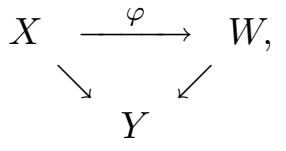

where

(1) $\bar{X}, \bar{Y}$ and $\bar{W}$ are equivariant completions of $X, Y$ and $W$, respectively,

(2) $\bar{X}$ is $\mathbb{Q}$-factorial, 
(3) $\bar{\varphi}: \bar{X} \rightarrow \bar{W}, \bar{X} \rightarrow \bar{Y}$ and $\bar{W} \rightarrow \bar{Y}$ are projective and

(4) $\rho(\bar{X} / \bar{W})=1$.

Thus, we fix an equivariant completion $\bar{\varphi}: \bar{X} \rightarrow \bar{W}$ of $\varphi$ as in Theorem 3.2. Let $\bar{\Delta}$ be the fan associated to $\bar{X}$ and $w \in \bar{\Delta}$ an $(n-1)$ dimensional cone such that $\bar{\varphi}(\mathrm{V}(w))$ is a point. Then, as in Section 2, we have an extremal primitive relation $a_{1} x_{1}+\cdots+a_{l} x_{l}=b_{1} y_{1}+\cdots+b_{m} y_{m}$ for $w$, where $x_{1}, \ldots, x_{l}, y_{1}, \ldots, y_{n} \in \mathrm{G}(\bar{\Delta})$. As in Section 2, we define $w^{\prime}:=\mathbb{R}_{\geq 0} y_{1}+\cdots+\mathbb{R}_{\geq 0} y_{m} \prec w$ and put $\sigma_{i} \in \bar{\Delta}$ be the cone generated by $\left\{x_{1}, \ldots, x_{l}\right\} \backslash\left\{x_{i}\right\}$ for $1 \leq i \leq l$.

LEMMA 3.3. $w^{\prime}+\sigma_{i} \in \Delta$ for any $1 \leq i \leq l$.

Proof. Obviously, $x_{1}, \ldots, x_{l}, y_{1}, \ldots, y_{m}$ are contained in the inverse image of a cone in $\Delta_{Y}$. So, by the properness of $f$, we complete the proof.

Thus, we call $a_{1} x_{1}+\cdots+a_{l} x_{l}=b_{1} y_{1}+\cdots+b_{m} y_{m}$ an extremal primitive relation in this case, too.

Proposition 2.4 also holds for this case. This immediately follows from the properness of $\varphi$ and Proposition 2.4. Though the setting is distinct from the one in Proposition 2.4, the statement is completely similar. We repeat it.

TheOREM 3.4. For any cone $\sigma \in \Delta$ such that $w^{\prime} \prec \sigma$, put $\sigma=w^{\prime}+$ $\sigma^{\prime}+\tau$, where $\sigma^{\prime}, \tau \in \Delta, \mathrm{G}\left(\sigma^{\prime}\right) \subset\left\{x_{1}, \ldots, x_{l}\right\}$ and $\mathrm{G}(\tau) \cap\left\{x_{1}, \ldots, x_{l}, y_{1}, \ldots\right.$, $\left.y_{m}\right\}=\emptyset$. Then, $w^{\prime}+\sigma_{i}+\tau \in \Delta$ for any $1 \leq i \leq l$.

In the remaining part of this section, we give an explicit combinatorial description for $\varphi: X \rightarrow W$ by using Theorem 3.4.

3.5. (Fano contractions) Suppose that $\varphi$ is a Fano contraction, that is, $\operatorname{dim} X>\operatorname{dim} W$. This condition is equivalent to $m=0$. Put $N^{\prime}:=$ $N /\left(\mathbb{Z} x_{1}+\cdots+\mathbb{Z} x_{l}\right)$. Then, we obtain the fan $\Delta_{W}$ in $N^{\prime}$ associated to $W$ by sending the cones in $\Delta$ through $N \rightarrow N^{\prime}$. The general fiber $F$ of $\varphi$ is the complete $\mathbb{Q}$-factorical toric variety of Picard number 1 whose extremal primitive relation is $a_{1} x_{1}+\cdots+a_{l} x_{l}=0$. In particular, if $\left\{x_{1}, \ldots, x_{l}\right\}$ generates the lattice, then $F \cong \mathbb{P}\left(a_{1}, \ldots, a_{l}\right)$ (see Example 2.8 and its front). 
3.6. (Birational contractions) Suppose that $\varphi$ is birational. This condition is equivalent to $m>0$. Put

$$
\widetilde{w}:=\mathbb{R}_{\geq 0} y_{1}+\cdots+\mathbb{R}_{\geq 0} y_{m}+\mathbb{R}_{\geq 0} x_{1}+\cdots+\mathbb{R}_{\geq 0} x_{l} .
$$

We remark that if $m=1$, then $y_{1} \in \mathbb{R}_{\geq 0} x_{1}+\cdots+\mathbb{R}_{\geq 0} x_{l}$. For $w^{\prime} \prec \sigma \in \Delta$, put $\sigma^{\prime}$ and $\tau$ as in Theorem 3.4. The fan $\Delta_{W}$ in $N$ associated to $W$ is as follows:

$$
\Delta_{W}=\left(\Delta \backslash\left\{\sigma \in \Delta \mid w^{\prime} \prec \sigma\right\}\right) \cup\left\{\widetilde{w}+\tau \mid w^{\prime} \prec \sigma=w^{\prime}+\sigma^{\prime}+\tau \in \Delta\right\} .
$$

The exceptional locus $A$ of $\varphi$ is $\mathrm{V}\left(w^{\prime}\right)$, while $B:=\varphi(A)=\mathrm{V}(\widetilde{w})$. We have $\operatorname{codim} A=m$ and $\operatorname{dim} B=n-l-m+1$. We note that $A$ is irreducible. This does not necessarily hold for non- $\mathbb{Q}$-factorial varieties (see [Fj2, Example 4.1]).

3.7. (Flips, flops and anti-flips) Suppose that $\varphi$ is a small contraction, that is, $\operatorname{codim} A \geq 2$. This condition is equivalent to $m \geq 2$. We use the same notation as above. Put

$$
w^{+}:=\mathbb{R}_{\geq 0} x_{1}+\cdots+\mathbb{R}_{\geq 0} x_{l} .
$$

Then, we obtain a fan $\Delta^{+}$in $N$ by star-subdividing $\Delta_{W}$ along $w^{+}$. Let $X^{+}$ be the associated toric variety. Then, the diagram

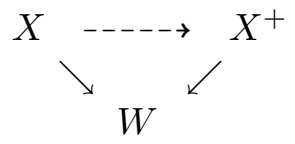

is

$$
\left\{\begin{array}{l}
\text { a flip } \\
\text { a flop } \\
\text { an anti-flip }
\end{array} \quad \text { if } a_{1}+\cdots+a_{l}-\left(b_{1}+\cdots+b_{m}\right)\left\{\begin{array}{l}
>0, \\
=0, \\
<0 .
\end{array}\right.\right.
$$

We remark that $b_{1} y_{1}+\cdots+b_{m} y_{m}=a_{1} x_{1}+\cdots+a_{l} x_{l}$ is an extremal primitive relation of $\Delta^{+}$.

\section{§4. Applications}

As applications of the results in the previous section, we can generalize some results in [Mu] (cf. [Fj1, Remark 3.3] and [Fj2, Theorem 3.13]). We use the same notation as in Sections 2 and 3. In addition, for the case of a Fano contraction, put $A:=X$ and $B:=W$. 
Let $f: X \rightarrow Y$ be a projective surjective toric morphism as in the previous sections. We assume that $X$ is smooth. For an extremal ray of $\mathrm{NE}(X / Y)$, let $\varphi_{R}: X \rightarrow W$ be the extremal contraction with respect to $R$. Then, the general fiber of $\varphi_{R}: A \rightarrow B$ is a projective space, since $X$ is smooth. So, let $C_{R}$ be a line in a general fiber of $A \rightarrow B$. The following is obvious.

LEMMA 4.1. $C_{R}$ spans $R$, and for any torus invariant prime divisor $D$ on $X$, we have $\left(D \cdot C_{R}\right) \leq 1$.

Remark 4.2. If $R$ contains a numerical equivalence class of torus invariant curves, then the local description of the extremal contraction $\varphi_{R}$ coincides with Reid's. Therefore, if $R$ contains a numerical equivalence class of torus invariant curves, then we can make $C_{R}$ torus invariant. This is obvious by Reid's description of $\varphi_{R}$.

Proposition 4.3. Let $f: X \rightarrow Y$ be as above, $L$ an line bundle on $X$ and $l$ a positive integer. Assume that $X$ is smooth. If $\left(L \cdot C_{R}\right) \geq l$ for every extremal ray $R$ of $\mathrm{NE}(X / Y)$, then for every torus invariant prime divisor $D$ on $X$, we have $\left(L(-D) \cdot C_{R}\right) \geq l-1$. In particular, if $L$ is $f$-ample, then $L(-D)$ is $f$-free.

Proof. The first part is obvious by Lemma 4.1. The last part follows from the equivalence of $f$-freeness and $f$-nefness on toric varieties.

Proposition 4.3 is a relative version of [Mu, Proposition 4.3 and Lemma 4.4].

Proposition 4.4. Let $f: X \rightarrow Y$ be as above and $L$ an $f$-ample line bundle on $X$. Assume that $X$ is smooth. For two distinct torus invariant prime divisors $D_{1}$ and $D_{2}$ on $X$, let $v_{1}$ and $v_{2}$ be the corresponding elements in $\mathrm{G}(\Delta)$, respectively. Then, $L\left(-D_{1}-D_{2}\right)$ is not $f$-free if and only if there exists an extremal ray $R$ of $\mathrm{NE}(X / Y)$ whose extremal primitive relation is $a_{1} x_{1}+\cdots+a_{l} x_{l}=b_{1} y_{1}+\cdots+b_{m} y_{m}$ such that $\left\{v_{1}, v_{2}\right\} \subset\left\{x_{1}, \ldots, x_{l}\right\}$ and $\left(L \cdot C_{R}\right)=1$.

Proof. If there exists an extremal ray $R$ as above, then $\left(L\left(-D_{1}-D_{2}\right)\right.$. $\left.C_{R}\right)=-1$. Therefore, $L\left(-D_{1}-D_{2}\right)$ is not $f$-nef, that is, not $f$-free.

So, suppose that $L\left(-D_{1}-D_{2}\right)$ is not $f$-nef. There exists an extremal ray $R$ of $\operatorname{NE}(X / Y)$ such that $\left(L\left(-D_{1}-D_{2}\right) \cdot C_{R}\right)<0$. Since $\left(L \cdot C_{R}\right) \geq 1$, 
$\left(D_{1} \cdot C_{R}\right) \leq 1$ and $\left(D_{2} \cdot C_{R}\right) \leq 1$, we have $\left(L \cdot C_{R}\right)=\left(D_{1} \cdot C_{R}\right)=\left(D_{2} \cdot C_{R}\right)=1$. This completes the proof by Proposition 2.7 .

Proposition 4.4 is the generalization of [Mu, Proposition 4.5], while the following is the one of [Mu, Proposition 4.6]. The proof is similar.

Proposition 4.5. Let $f: X \rightarrow Y$ be as above and $L$ an $f$-ample line bundle on $X$. Assume that $X$ is smooth. For a torus invariant prime divisor $D$ on $X$, let $v$ be the corresponding element in $\mathrm{G}(\Delta)$. Then, $L(-D)$ is not $f$-ample if and only if there exists an extremal ray $R$ of $\mathrm{NE}(X / Y)$ whose extremal primitive relation is $a_{1} x_{1}+\cdots+a_{l} x_{l}=b_{1} y_{1}+\cdots+b_{m} y_{m}$ such that $v \in\left\{x_{1}, \ldots, x_{l}\right\}$ and $\left(L \cdot C_{R}\right)=1$.

\section{REFERENCES}

[B] V. Batyrev, On the classification of smooth projective toric varieties, Tohoku Math. J., 43 (1991), 569-585.

[C] C. Casagrande, Contractible classes in toric varieties, Math. Z., 243 (2003), 99-126.

[Fj1] O. Fujino, Notes on toric varieties from Mori theoretic viewpoint, Tohoku Math. J., 55 (2003), 551-564.

[Fj2] O. Fujino, Equivariant completions of toric contraction morphisms, preprint, math.AG/0311068, to appear in Tohoku Math. J.

[FS] O. Fujino and H. Sato, Introduction to the toric Mori theory, Mich. Math. J., 52 (2004), 649-665.

[Fl] W. Fulton, Introduction to toric varieties, Annals of Mathematics Studies, 131, The William H. Roever Lectures in Geometry, Princeton University Press, Princeton, NJ, 1993.

[K] A. Kasprzyk, Toric Fano 3-folds with terminal singularities, preprint, math.AG/ 0311284, to appear in Tohoku Math. J.

[Ma] K. Matsuki, Introduction to the Mori program, Universitext, Springer-Verlag, New York, 2002.

[Mu] M. Mustaţă, Vanishing theorems on toric varieties, Tohoku Math. J., 54 (2002), 451-470.

[O] T. Oda, Convex bodies and algebraic geometry, An introduction to the theory of toric varieties, Translated from the Japanese, Ergebnisse der Mathematik und ihrer Grenzgebiete (3) [Results in Mathematics and Related Areas (3)] 15, Springer-Verlag, Berlin, 1988.

[R] M. Reid, Decomposition of toric morphisms, Arithmetic and geometry, Vol. II, Progr. Math., 36, Birkhäuser Boston, MA (1983), pp. 395-418.

[S] H. Sato, Toward the classification of higher-dimensional toric Fano varieties, Tohoku Math. J., 52 (2000), 383-413. 
Department of Mathematics

Tokyo Institute of Technology 2-12-1 Oh-Okayama

Meguro-ku

Tokyo, 152-8551

Japan

hirosato@math.titech.ac.jp 